\title{
ESTUDIO BIBLIOMÉTRICO DEL SECTOR PROFESIONAL DE LAS RELACIONES PÚBLICAS Y LA COMUNICACIÓN CORPORATIVA CON PERSPECTIVA DE GÉNERO EN LAS REVISTAS INDEXADAS EN WEB OF SCIENCE CORE COLLECTION, SCOPUS Y PROQUEST CENTRAL (1992-2014)
}

\author{
BIBLIOMETRIC STUDY OF THE PROFESSIONAL SECTOR OF \\ PUBLIC RELATIONS AND CORPORATE COMMUNICATION \\ WITH GENDER PERSPECTIVE IN JOURNALS INDEXED \\ IN WEB OF SCIENCE CORE COLLECTION, SCOPUS AND \\ PROQUEST CENTRAL (1992-2014)
}

\author{
Susana MIQUEL-SEGARRA \\ Universitat Jaume I de Castelló \\ Marián NAVARRO-BELTRÁ \\ Universidad Católica de Murcia \\ Marta MARTíN-LLAGUNO \\ Universidad de Alicante
}

Recibido: 12/01/2016

Aceptado: 28/04/ 2016

\section{Resumen}

Los trabajos que emplean la perspectiva de género para el estudio de los profesionales de la comunicación corporativa y las relaciones públicas no han sido sistematizados hasta la fecha. Por tanto, el objetivo de este artículo es compilar, revisar, sintetizar y examinar la producción científica relacionada con el entorno laboral de las relaciones públicas o de la comunicación corporativa con enfoque de género. Para ello se ha realizado una revisión sistemática. El universo de estudio ha estado conformado por los 
artículos publicados en inglés o en español en revistas indexadas en las bases de datos Web of Science Core Collection, Scopus o Proquest Central (área temática de Ciencias Sociales) entre 1992 y 2014. Los principales resultados de la investigación apuntan a un reciente, aunque discreto, interés por el estudio de este ámbito laboral en diversas zonas geográficas. Además, en la mayoría de los textos se llega a la conclusión de que en las estructuras y en los procesos de las relaciones públicas y la comunicación corporativa se produce algún tipo de discriminación.

Palabras clave: perspectiva de género, práctica profesional, relaciones públicas, comunicación corporativa, revistas científicas

\begin{abstract}
The academic works that employ a gender perspective to the study of corporative communication and public relation professionals have not been systematized to date. Thus, the aim of this paper is to compile, review, synthesize and examine the scientific production related to the working environment of public relations or communication management with a gender perspective. For that, we did a systematic review. The study group has been formed by the articles published in English or Spanish in journals indexed in Web of Science Core Collection, Scopus and Proquest Central (Thematic Social Sciences) between 1992 and 2014. The main results of the research point to a recent, though discreet, interest in the study of this workplace in different geographical areas. Moreover, the most of the texts conclude that in the structures and in the processes of public relations and communication management some discrimination occurs.
\end{abstract}

Keywords: gender perspective; professional practice; public relations; communication management; scientific journals 


\section{INTRODUCCIÓN}

Desde que la Declaración Universal de los Derechos Humanos (Naciones Unidas 1948) trazara el planteamiento universalista de la igualdad entre hombres y mujeres, múltiples declaraciones han apostado por erradicar el sexismo en la sociedad ${ }^{1}$ contemplando, cada vez más a menudo, de manera directa o indirecta, el papel de la comunicación ${ }^{2}$ por ser un agente de socialización fundamental (Messineo 752; Tsai 423). Así, la eliminación de la desigualdad por razón de sexo en la sociedad de la información en general, y en su comunicación pública en particular, no sólo se ha convertido en un tema de preocupación política, sino también académica (Eisend; Gentry, Harrison; Martín-Llaguno, Navarro-Beltrá; Navarro-Beltrá, Martín-Llaguno 2012; Navarro-Beltrá, Martín-Llaguno 2013).

Casi desde sus inicios, los trabajos de las ciencias de la comunicación contemplaron el sexo como categoría de análisis (Hovland, Lumsdaine, Sheffield; Lasswell; Lazarsfeld, Berelson, Gaudet). Sin embargo, fueron las investigaciones feministas, que aportaron el concepto de género, las que inicialmente se preocuparon por las desigualdades entre hombres y mujeres y por la perpetuación de las estructuras de poder sexistas (Van Zoonen 15).

En tiempos recientes, la perspectiva o el enfoque de género -que implica tomar en consideración y prestar atención a las diferencias entre hombres y mujeres en cualquier ámbito (Comisión de las Comunidades Europeas; Comisión Europea) - se aplica al análisis de la comunicación, en cualquiera de

1. A modo de ejemplo: Declaración sobre la Eliminación de la Discriminación contra la Mujer (Naciones Unidas 1967); Proclamación de Teherán (Naciones Unidas 1968), Convención sobre la Eliminación de Todas las Formas de Discriminación contra la Mujer (Naciones Unidas 1979); Carta de los Derechos Fundamentales de la Unión Europea (Unión Europea 2000); Versiones Consolidadas del Tratado de la Unión Europea y del Tratado Constitutivo de la Comunidad Europea (Unión Europea 2006).

2. A modo de ejemplo: Resolución sobre el Tratamiento de la Imagen de las Mujeres y de los Hombres en la Publicidad y en los Medios de Comunicación (Unión Europea 1995); Resolución sobre la Discriminación de la Mujer en la Publicidad (Unión Europea 1997); Informe sobre el Impacto del Marketing y la Publicidad en la Igualdad entre Mujeres y Hombres (Comisión de Derechos de la Mujer e Igualdad de Género del Parlamento Europeo 2008). 
sus dimensiones -información (Desmond, Danilewicz; Macharia, O'Connor, Ndangam), persuasión (Döring, Pöschl; Espinar; Gil; Valls-Fernández, Martínez-Vicente) y entretenimiento (Espinar; Gil; Lauzen, Dozier)-; en cualquiera de sus ámbitos -público y privado- y con cualquiera de sus tecnologías.

La academia se ha preocupado mayoritariamente por la representación estereotipada de la población femenina en los discursos, especialmente el informativo y el persuasivo (Dominick, Rauch; Eisend; Furnham, Paltzer; Gallego, Altés, Melús, Soriano, Cantón; Silverstein, Silverstein). En este sentido, resulta necesario analizar el sexismo no sólo en los mensajes, sino también en la recepción y en las estructuras. Así, parece urgente diversificar la investigación en comunicación con perspectiva de género y aplicarla también a los sistemas y procesos laborales, potenciando nuevas líneas de trabajo. En especial, diversas recomendaciones animan el uso y el desarrollo de indicadores en este ámbito (UNESCO).

El análisis de los sistemas, de las estructuras y de los procesos en el sector de la comunicación con este enfoque ha sido objeto incipiente de interés en la última década en España. Las investigaciones que se están empezando a realizar con perspectiva de género en este país sobre los profesionales de la comunicación corporativa y los directores de comunicación (Martín-Llaguno, Miquel Segarra, Navarro-Beltrá), se unen a los estudios realizados desde 2003 por la Asociación de Directivos de Comunicación (Dircom) ${ }^{3}$ y a los efectuados desde 2007 por Euprera y la European Association of Communication Directors $^{4}$ que tratan de ampliar el conocimiento existente sobre los profesionales de las relaciones públicas y la comunicación corporativa. Por su parte, los trabajos sobre las agencias de publicidad y los publicitarios desarrollados a partir de 2007 en España (Hernández-Ruiz, Martín-Llaguno, Beléndez-Vázquez; Martín-Llaguno, Beléndez-Vázquez, Hernández-Ruiz; Martín-Llaguno 2007a; Martín-Llaguno 2007b), se unen a los estudios impulsados por el Institute of Practitioners in Advertising (IPA) del Reino Unido en la década de los años 90 del siglo XX (Klein) y a la más extensa investigación sobre la

3. Dircom es una asociación profesional española que se creó en 1992 y que agrupa a los directivos y a los profesionales de la comunicación de las empresas, instituciones y consultoras en España. Posee más de 950 socios y cuenta con 7 delegaciones territoriales. Entre las publicaciones impulsadas por esta asociación destacan los Anuarios de la Comunicación, estudios que se realizan con periodicidad anual. Para más información ver: http://www.dircom.org/, consultado el 12-04-2016.

4. El European Communication Monitor (ECM) es un estudio que se realiza anualmente en Europa sobre el ámbito de la comunicación estratégica y las relaciones públicas. Para más información ver: http://www.euprera.org/?p=30, consultado el 12-04-2016. 
situación en función de sexos de la profesión periodística (Arnold; Arnold, Linton; Arnold, Lozada, Linton; Bowman; Falk, Grizard).

Sin embargo, quedan ámbitos de trabajo de la comunicación todavía por explorar, al menos en España. Pese a su importancia en la confección del discurso de la Sociedad de la Información y el Conocimiento (SIC) y su creciente importancia como sector (International Communications Consultancy Organisation-ICCO-), la situación laboral de los profesionales de la comunicación corporativa y las relaciones públicas apenas ha acaparado la atención de los investigadores, especialmente si consideramos la perspectiva de género.

Y eso que, al margen de la práctica profesional, estos ámbitos han concitado un campo propio de investigación (Botan, Taylor) que ha sido sintetizado en sucesivas revisiones sistemáticas sobre la disciplina de las relaciones públicas en general (Castillo, Xifra; Kim, Choi, Reber, Kim; Krohling; Míguez-González, Baamonde-Silva, Corbacho-Valencia; Morton, Lin; Pasadeos, Berger, Renfro; Pasadeos, Renfro, Hanily; Pasadeos, Renfro), o sobre prácticas específicas como la comunicación de crisis (García, Smolak-Lozano; Kim, Avery, Lariscy), la Responsabilidad Social Corporativa (Golob, Podnar, Elving, Nielsen, Thomsen, Schultz), la relación de la organización con sus públicos (Ki, Shin) y sus teorías (Sallot, Lyon, Acosta-Alzuru, Jones) y las relaciones públicas on-line (Ye, $\mathrm{Ki}$ ). Cabe señalar que también se han realizado estudios bibliométricos en España sobre el ámbito de la comunicación en general (Castillo, Carretón; De-Filippo), y de la publicidad (Marcos, Martínez, Blasco; Martínez) y del periodismo (Oliva) en particular, con el propósito de ampliar el conocimiento existente sobre estos temas.

Los trabajos que emplean la perspectiva de género para el estudio de los profesionales de la comunicación corporativa y las relaciones públicas no han sido sistematizados hasta la fecha. Por tanto, resulta necesario subsanar esta carencia habida cuenta que diversos autores apuntan a que las relaciones públicas y la comunicación corporativa pueden ser ámbitos laborales especialmente feminizados (Aldoory 670; Chen 431; Sha, Toth 93; Simorangkir 26; Wrigley 32).

De esta forma, el objetivo de este artículo es compilar, revisar, sintetizar y examinar una muestra de la producción científica existente relacionada con el entorno laboral de las relaciones públicas o de la comunicación corporativa con enfoque de género (es decir, que toma en consideración y/o presta atención a las diferencias entre hombres y mujeres). De manera más concreta se pretende: 
- Conocer las características de producción de este tipo de textos, entre otras, los años, las revistas, los países, los autores y las instituciones más prolíficas.

- Examinar la calidad científica de estos documentos en función del número de citas que reciben y analizar la calidad de las revistas que publican estas investigaciones según sus índices de impacto.

- Describir las variables investigadas: tema, objeto de estudio y ámbito geográfico.

- Referir la metodología empleada.

- Descubrir las particularidades de la bibliografía utilizada: número de textos que conforman las referencias, idioma de esos documentos y escritos más citados.

\section{METODOLOGÍA}

Con el propósito de alcanzar los objetivos señalados anteriormente se ha realizado una revisión sistemática de la literatura científica, con criterios bibliográficos, de modo que el universo de estudio ha estado conformado por los artículos publicados en inglés o en español en revistas indexadas en las bases de datos Web of Science Core Collection, Scopus o Proquest Central (área temática de Ciencias Sociales) entre 1992 y junio de 2014.

Web of Science Core Collection y Scopus han sido seleccionadas por ser las bases de mayor relevancia a nivel mundial. Sin embargo, con relación a nuestro tema de estudio, estas bases de datos poseen un importante sesgo temático: es más habitual que indexen revistas relacionadas con el ámbito de las Ciencias que con el de las Ciencias Sociales. Por tanto, para tratar de solventar este problema, también se ha analizado el área temática de Ciencias Sociales de la base de datos Proquest Central.

Por su parte, el periodo temporal señalado anteriormente ha sido escogido porque abarca desde la creación de la Asociación de Directivos de Comunicación (Dircom) $)^{5}$ en España hasta el momento en el que se llevó a cabo la recogida de datos (junio de 2014). Inicialmente se han seleccionado todos los textos que respondieron a las ecuaciones que se muestran en la tabla 1.

5. La creación de esta institución es importante debido a que el asociacionismo favorece la elaboración de investigaciones relacionadas con el sector. 
Tabla 1: ecuaciones de búsqueda utilizadas

\begin{tabular}{|l|l|}
\hline $\begin{array}{c}\text { Bases de } \\
\text { datos }\end{array}$ & \multicolumn{1}{c|}{ Ecuaciones de búsqueda } \\
\hline Scopus & $\begin{array}{l}\text { Año de publicación: } 1992 \text { - to - present } \\
\text { Tipo de fuente: ALL } \\
\text { Subject areas: Todas (Life Sciences, Health Sciences, Physical Sciences, } \\
\text { Social Sciences \& Humanities) } \\
\text { Campo: Article title/abstract/keywords } \\
\text { Ecuación: «human capital» OR labor* OR work* AND «public relations» } \\
\text { OR PR OR «communication manager» OR «corporate communication» } \\
\text { AND «glass ceiling» OR «sticky floor» OR gender OR sex* OR «work- } \\
\text { family conflict» OR «family-work conflict» (article title, abstract, } \\
\text { keywords) }\end{array}$ \\
\hline Proquest & $\begin{array}{l}\text { Año de publicación: Posterior a - diciembre/31/1991 } \\
\text { Tipo de fuente: revistas científicas } \\
\text { Subject areas: Ciencias Sociales } \\
\text { Document type:: artículo, artículo principal y reseña/revisión } \\
\text { Campo: Resumen/ Título/ Encabezamiento de materia -todos-: } \\
\text { Ecuación: («human capital» OR labour* OR labor* OR work*) AND } \\
\text { («public relations» OR PR OR «communication manager» OR «corporate } \\
\text { communication») AND ( «glass ceiling» OR «sticky floor» OR gender OR } \\
\text { sex* OR «work-family conflict» OR «family-work conflict») }\end{array}$ \\
\hline $\begin{array}{l}\text { Web of } \\
\text { Science } \\
\text { Core } \\
\text { Collection }\end{array}$ & $\begin{array}{l}\text { Año de publicación: 1992-2002 OR 2003-2013 OR 2014 } \\
\text { Tipo de fuente: ALL } \\
\text { Campo: Tema } \\
\text { Ecuación: («human capital» OR labour* OR labor* OR work*) AND } \\
\text { («public relations» OR PR OR «communication manager» OR «corporate } \\
\text { communication») AND ( «glass ceiling» OR «sticky floor» OR gender OR } \\
\text { sex* OR «work-family conflict» OR «family-work conflict») }\end{array}$ \\
\hline
\end{tabular}

Así, se han recuperado los artículos en cuyo «título», «resumen» y/o «palabras clave» aparecieran uno o varios de los términos escogidos. Estas palabras clave, que se pueden observar en la tabla 1, fueron seleccionadas por identificar tres ámbitos de estudio esenciales para la investigación: comunicación, trabajo y sexo. Dado que nuestro interés estaba en obtener textos que analizaran el entorno laboral de la comunicación corporativa o las relaciones públicas con enfoque de género, además de «gender» y «sex», se decidió incluir vocablos específicos referidos a la discriminación sexual en el trabajo que se han abordado en otros campos de la comunicación, a saber: "glass ceiling» (García-González, Piñeiro-Otero; Martín-Llaguno 2007a; Martín-Llaguno 2007b); «sticky floor» (Martín-Llaguno 2007a; Martín-Llaguno 2007b); «work-family conflict» y «family-work conflict» (Kelly et al.; Major, Klein, Ehrhart; Martín-Llaguno, Baquerín de Riccitelli; Martín-Llaguno, BeléndezVázquez, Hernández-Ruiz). 
Una vez extraído el listado de artículos que respondían a los parámetros definidos con anterioridad a la realización de la búsqueda, y a partir de la lectura del título, del resumen y/o del texto completo, dos investigadores han seleccionado aquéllos que se ajustan al objeto de investigación (abordaje empírico o teórico del ámbito laboral de las relaciones públicas y la comunicación corporativa y/o de los directores de comunicación con enfoque de género).

La lista de los documentos finalmente analizados se puede observar en la tabla 2. Se ha considerado que han cumplido los criterios de inclusión 29 artículos, un 56\% de los cuales aparecían indexados simultáneamente en más de una base de datos, dado que Web of Science Core Collection y Scopus incluían 16 textos respectivamente y Proquest 15. El proceso de selección de artículos sintetizado se muestra en la Figura 1.

Tabla 2: artículos analizados

\begin{tabular}{|c|}
\hline Artículos analizados \\
\hline $\begin{array}{l}\text { ALDOORY, Linda. «(Re)Conceived Feminist Paradigm for Public Relations: A Case for } \\
\text { Substantial Improvement». Journal of Communication } 55.4 \text { (2005): 668-684. }\end{array}$ \\
\hline $\begin{array}{l}\text { AL-JENAIBI, Badreya. "Gender Issues in the Diversity and Practice of Public Relations in } \\
\text { the UAE Case Study of P.R. Male Managers and Female P.R. Practitioners». International } \\
\text { Journal of E-Politics } 2.3 \text { (2011): 35-56. }\end{array}$ \\
\hline $\begin{array}{l}\text { Austin, Lucinda L. «Framing Diversity: A Qualitative Content Analysis of Public } \\
\text { Relations Industry Publications». Public Relations Review } 36.3 \text { (2010): 298-301. }\end{array}$ \\
\hline $\begin{array}{l}\text { BEURER-ZUELLIG, Bettina, Christian FIESELER y Miriam MECKEL. «A Descriptive Inquiry } \\
\text { Into the Corporate Communication Profession in Europe». Public Relations Review } 35.3 \\
\text { (2009): 270-279. }\end{array}$ \\
\hline $\begin{array}{l}\text { BOVET, Susan Fry. «Sexual Harassment: What's Happening and how to Deal with it». The } \\
\text { Public Relations Journal } 49.11 \text { (1993): } 26 \text {. }\end{array}$ \\
\hline $\begin{array}{l}\text { CHEN, Yi-Ning Katherine. «Social Capital, Human Capital, and Career Success in Public } \\
\text { Relations in Taiwan». Chinese Journal of Communication } 4.4 \text { (2011): 430-449. }\end{array}$ \\
\hline $\begin{array}{l}\text { CREEDON, Pamela J. «Acknowledging the Infrasystem: A Critical Feminist Analysis of } \\
\text { Systems Theory». Public Relations Review } 19.2 \text { (1993): 157-166. }\end{array}$ \\
\hline $\begin{array}{l}\text { ELVING, Wim, Betteke VAN RULER, Michael GOODMAN y Christina GENEST. } \\
\text { «Communication Management in the Netherlands: Trends, Developments, and } \\
\text { Benchmark with US Study». Journal of Communication Management } 16.2 \text { (2012): } \\
\text { 112-132. }\end{array}$ \\
\hline $\begin{array}{l}\text { FIELDEN, Sandra L., Ralph TENCH y Johanna FAWKES. «Freelance Communications } \\
\text { Workers in the UK: The Impact of Gender on Well-being». Corporate Communications } \\
8.3 \text { (2003): 187-196. }\end{array}$ \\
\hline
\end{tabular}


FröHLICH, Romy y Sonja B.PeTERS. «PR Bunnies Caught in the Agency Ghetto? Gender Stereotypes, Organizational Factors, and Women's Careers in PR Agencies». Journal of Public Relations Research 19.3 (2007): 229-254.

Gallicano, Tiffany Derville, Patricia CurTin y Kelly Matthews. «I Love what I do, but... A Relationship Management Survey of Millennial Generation Public Relations Agency Employees». Journal of Public Relations Research 24.3 (2012): 222-242.

HAZLETON, Vicent y Bey-Ling SHA. «Generalizing from PRSA to Public Relations: How to Accommodate Sampling Bias in Public Relations Scholarship». Public Relations Review 38.3 (2012): 438-445.

HORSLEY, J. Suzanne. «Women's Contributions to American Public Relations, 19401970». Journal of Communication Management 13. 2 (2009): 100-115.

JiANG, Hua y Hongmei SHEN. «Toward a Theory of Public Relations Practitioners' Own Conflict: Work Versus Life». Journal of Public Relations Research 25.3 (2013): 259-279.

KeRn-Foxworth, Marilyn, Oscar GANDY, Barbara Hines y Debra A. Miller. «Assessing the Managerial Roles of Black Female Public Relations Practitioners using Individual and Organizational Discriminants». Journal of Black Studies 24.4 (1994): 416-434.

LAUZEN, Martha. «Effects of Gender on Professional Encroachment in Public Relations». Journalism Quarterly 69.1 (1992): 173-180.

PAnigyrakis, George G. y Cleopatra A. Veloutsou. «Sex-Related Differences of Public Relations Managers in Consumer Goods Companies in Greece and Italy». Gender in Management: An International Journal 13.2 (1998): 72-82.

POMPPER, Donnalyn. «The Gender-Ethnicity Construct in Public Relations Organizations: Using Feminist Standpoint Theory to Discover Latinas' Realities». Howard Journal of Communications 18.4 (2007): 291-311.

POMPPER, Donnalyn. «On Social Capital and Diversity in a Feminized Industry: Further Developing a Theory of Internal Public Relations». Journal of Public Relations Research 24.1 (2012): 86-103.

POMPPER, Donnalyn y Taejin JUNG. «'Outnumbered Yet Still on Top, but for how Long?' Theorizing about Men Working in the Feminized Field of Public Relations». Public Relations Review 39.5 (2013): 497-506.

RYAN, Michael. «Public Relations and the Web: Organizational Problems, Gender, and Institution Type». Public Relations Review 29.3 (2003): 335-349.

SAllot, Lynne M., Glen T. CAMERON y Ruth Ann WeAVER LARISCY. «Pluralistic Ignorance and Professional Standards: Underestimating Professionalism of our Peers in Public Relations». Public Relations Review 24.1 (1998): 1-19.

SCOTT, Denise Benoit. «The Costs and Benefits of Women's Family Ties in Occupational Context: Women in Corporate-Government Affairs Management». Community, Work \& Family 4.1 (2001): 5-27.

SHA, Bey-Ling y Elizabeth L. TотH. «Future Professionals' Perceptions of Work, Life, and Gender Issues in Public Relations». Public Relations Review 31.1 (2005): 93-99.

SIMORANGKIR, Deborah. "The Impact of the Feminization of the Public Relations Industry in Indonesia on Communication Practice». International Journal of Strategic Communication 5.1 (2011): 26-48. 
TINDALl, Natalie T. J. y Richard D. WATERS. «Coming Out to Tell our Stories: Using Queer Theory to Understand the Career Experiences of Gay Men in Public Relations». Journal of Public Relations Research 24.5 (2012): 451-475.

VANC, Antoaneta y Candace WHITE. «Cultural Perceptions of Public Relations Gender Roles in Romania». Public Relations Review 37.1 (2011): 103-105.

WOLF, Katharina. «PR Career Progression - the Gap between Traditional Research and the UK Industry's Perception». Journal of Communication Management 10.2 (2006): 174-190.

Wrigley, Brenda J. «Glass Ceiling? what Glass Ceiling? A Qualitative Study of how Women View the Glass Ceiling in Public Relations and Communications Management». Journal of Public Relations Research 14.1 (2002): 27-55.

Figura 1: proceso de selección de artículos

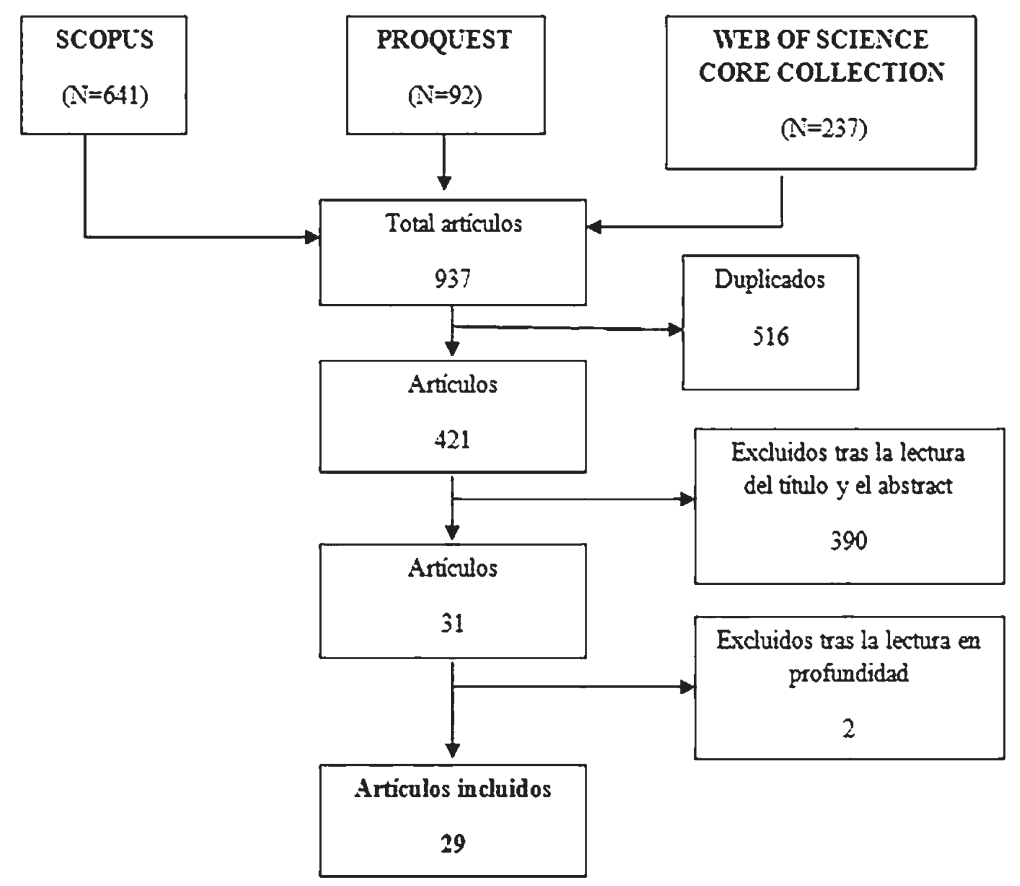

El texto completo de los 29 artículos finalmente seleccionados se ha analizado y codificado con base en un protocolo de 55 variables agrupadas en cinco categorías: a) características del artículo, b) autores y organizaciones, c) contenido de los textos, d) características de la revista y e) calidad de los escritos (índice de impacto de la revista en el año de divulgación del artículo y número de citas del texto). 
En el proceso de codificación han trabajado dos personas durante tres meses y entre ellas han obtenido un índice medio de Kappa del 0,875. Para analizar los artículos se ha creado una base de datos en el programa informático SPSS versión 15. En concreto, se han utilizado las frecuencias, las tablas de respuesta múltiple, los gráficos de líneas y las tablas de contingencia.

\section{RESULTADOS}

\subsection{Productividad}

Como se puede observar en la Figura 2, la producción científica que aplica el enfoque de género al ámbito de estudio seleccionado se ha ido incrementando a lo largo del tiempo, de manera que, entre 1992 y 2010, aparecen, como máximo, 2 escritos por año, mientras que, entre 2011 y 2012 (el periodo más prolífico) se publican una media de 9 artículos anuales. El descenso entre 2013 y 2014 puede ser explicado en parte por la demora en la inclusión de textos en las bases de datos.

Figura 2: año de publicación de los artículos

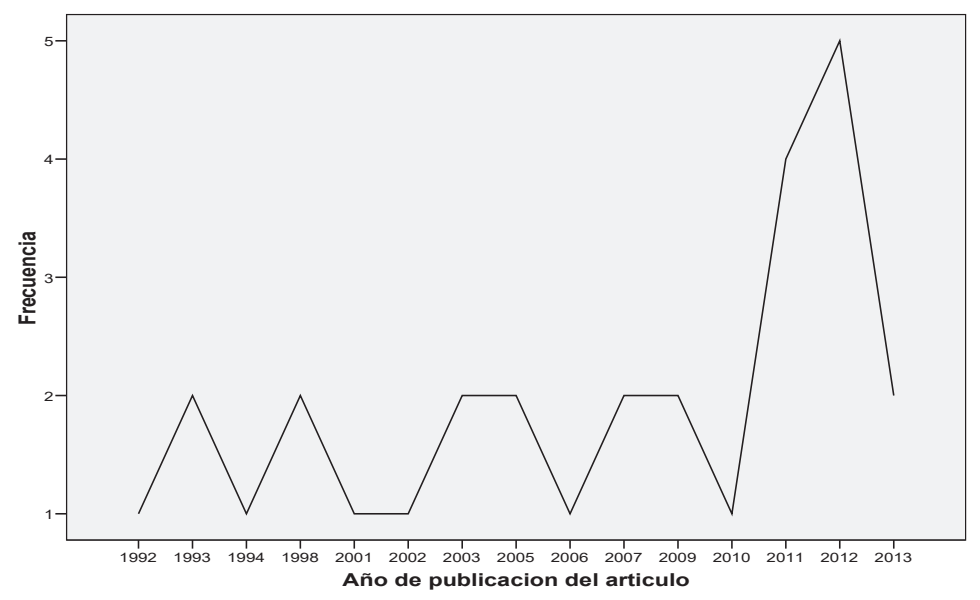

Los 29 documentos examinados han sido encontrados en 14 revistas científicas diferentes, de las cuales 11 sólo han publicado un artículo. Las cabeceras que más atención han prestado al tema, por este orden, han sido Public Relations Review (con 9 textos que suponen un 31\% del total), Journal of Public

6. Índice calculado para aquellas variables que requieren un juicio por parte del investigador. 
Relations Research (con 6, el 20,7\%) y Journal of Communication Management (con 3, el 10,3\%). La mayoría de revistas que han tocado nuestro tema se centran en al ámbito de las relaciones públicas, la comunicación corporativa, la dirección en comunicación o el management. Cabe destacar que ninguna revista especializada en género ha publicado estudios específicos sobre el ámbito de la comunicación corporativa o las relaciones públicas.

Por otro lado, el $72 \%$ de las 14 revistas son de Estados Unidos y el 28\% de Reino Unido, dos naciones de ámbito anglosajón autoras de la mayor producción científica en general. Sin embargo, se han encontrado textos procedentes de 10 países (Estados Unidos, Emiratos Árabes Unidos, Suiza, Taiwán, Países Bajos, Reino Unido, Alemania, Grecia, Indonesia y Australia), lo que da cuenta de que el reciente, aunque discreto, interés en el estudio del ámbito laboral de los profesionales de la comunicación corporativa y las relaciones públicas con perspectiva de género, se produce en diversas zonas geográficas.

Más de la mitad de los trabajos $(51,7 \%)$ tienen un único firmante, de modo que el índice de coautoría no supera el 1,76. En este mismo sentido vemos cómo el 27,6\% de los textos son rubricados por dos personas, el 13,8\% por tres y únicamente el $6,9 \%$ (dos escritos) cuenta con más de tres autores. Solamente dos de los 48 signatarios que aparecen firman más de un artículo: Donnalyn Pompper se puede observar en 3 ( 2 publicados en solitario y 1 en coautoría) y Bey-Ling Sha en 2 (ambos como coautora).

El tema parece interesar más a mujeres que a hombres: en todos los textos menos uno aparece al menos una autora mientras que en 19 artículos no hay firmantes masculinos (ver tabla 3). La feminización sobre este tipo de investigaciones se confirma al comprobar que la mayoría de las primeras autoras son mujeres $(86,2 \%)$, situación que está en consonancia con la feminización del ámbito de las relaciones públicas (Chen 431), ya que este ha sido considerado un campo de trabajo femenino (Simorangkir 26).

Tabla 3: número de firmantes por sexo

\begin{tabular}{|l|c|c||c|c|c|}
\hline $\begin{array}{c}\text { Número de } \\
\text { firmantes: } \\
\text { mujeres }\end{array}$ & Frecuencia & Porcentaje & $\begin{array}{c}\text { Número de } \\
\text { firmantes: } \\
\text { hombres }\end{array}$ & Frecuencia & Porcentaje \\
\hline Cero & 1 & 3,4 & Cero & 19 & 65,5 \\
\hline Uno & 18 & 62,1 & Uno & 9 & 31 \\
\hline Dos & 8 & 27,6 & Dos & 1 & 3,4 \\
\hline Tres & 2 & 6,9 & Tres & - & - \\
\hline TOTAL & 29 & 100 & TOTAL & 29 & 100 \\
\hline
\end{tabular}


Para completar la descripción de las características de este tipo de producción científica, cabe mencionar que en la elaboración de estos estudios las relaciones interinstitucionales no han sido frecuentes, ya que la mayoría de los artículos han sido realizados por investigadores pertenecientes a una misma universidad $(62,1 \%)$.

La Universidad Estatal de San Diego ha sido la institución más prolífica en el análisis de este tipo de cuestiones, seguida de la Universidad de Maryland y la Universidad de Temple. Otras universidades desde las que se han abordado con cierta frecuencia estos temas son la Universidad de Oregon, la Universidad de Georgia, la Universidad de St. Gallen, la Universidad Metropolitana de Leeds, la Baruch College de la Ciudad Universitaria de Nueva York, la Universidad de Amsterdam y la Universidad de Múnich Ludwig-MaximiliansUniversität. La dispersión de la investigación es grande y es difícil decir que existe un centro de referencia. Sí lo hay en cuanto a país, EE.UU, donde se ubican 28 de los autores (58,33\%). Reino Unido y Grecia aportan cada uno de ellos cuatro investigadores. Tres de los firmantes son de Suiza y dos de los Países Bajos y Alemania respectivamente. Por su parte, los Emiratos Árabes Unidos, Taiwán, Indonesia y Australia únicamente contribuyen al estudio de esta temática con un autor.

\subsection{Calidad de los artículos y de las revistas}

El 58,6\% de los textos analizados se publicó en revistas que se encontraban indexadas en la base de datos Journal Citation Reports (JCR) el año en el que fueron divulgados. Sin embargo, estas revistas aparecían principalmente en los cuartiles de menor prestigio: en el 60\% de los casos en el Q3, en el 20\% en el Q4, en el 15\% en el Q2 y únicamente el 5\% estaba en el Q1. La valoración de la calidad mejora ligeramente si se utiliza el SCImago Journal \& Country Rank (SJR) en lugar del JCR. Así, de los 23 textos que fueron publicados a partir de 1999 (año desde el que se encuentra disponible esta base de datos), 15 formaban parte de las revistas consideradas por SJR en el año de su publicación, que en un 37,8\% estaban catalogadas en el Q1, en otro 37,8\% en el Q2, en un 21,6\% en el Q3 y únicamente en un 2,7\% en el Q4.

El 59,3\% de los textos en JCR están considerados bajo la categoría «communication» y el 33,3\% pertenece a «business». Lo mismo sucede con los textos indexados en SJR, que en un 29,7\% de las ocasiones también se catalogan en "communication», aunque en este caso un 19\% de los textos respectivamente se incluyen bajo las categorías de «organizational behavior and human resource management» $\mathrm{y}$ «marketing» (ver tabla 4). 
Tabla 4: artículos en función de las categorías a las que pertenecen las revistas en JCR y SJR

\begin{tabular}{|l|c|c|c|}
\hline \multirow{2}{*}{ Categoría JCR } & \multicolumn{2}{c|}{ Respuestas } & \multirow{2}{*}{$\begin{array}{c}\text { Porcentaje de } \\
\text { casos }\end{array}$} \\
\cline { 2 - 3 } & Frecuencia & Porcentaje & 94,1 \\
\hline Communication & 16 & 59,3 & 52,9 \\
\hline Business & 9 & 33,3 & 5,9 \\
\hline Social science interdisciplinary & 1 & 3,7 & 5,9 \\
\hline Ethnic studies & 1 & 3,7 & $\mathbf{1 5 8 , 8}$ \\
\hline TOTAL JCR & $\mathbf{2 7}$ & $\mathbf{1 0 0}$ & \\
\hline
\end{tabular}

\begin{tabular}{|l|c|c|c|}
\hline \multirow{2}{*}{ Categoría SJR } & \multicolumn{2}{c|}{ Respuestas } & \multirow{2}{*}{$\begin{array}{c}\text { Porcentaje de } \\
\text { casos }\end{array}$} \\
\cline { 2 - 3 } & Frecuencia & Porcentaje & 73,3 \\
\hline Communication & 11 & 29,7 & 46,7 \\
\hline Marketing & 7 & 18,9 & 46,7 \\
\hline $\begin{array}{l}\text { Organizational behavior and } \\
\text { human resource management }\end{array}$ & 7 & 18,9 & 33,3 \\
\hline Sociology and political science & 5 & 13,5 & 26,7 \\
\hline Public administration & 4 & 10,8 & 6,7 \\
\hline Language and linguistics & 1 & 2,7 & 6,7 \\
\hline Linguistics and language & 1 & 2,7 & 6,7 \\
\hline Strategy and management & 1 & 2,7 & 246,7 \\
\hline TOTAL & 37 & 100 & \\
\hline
\end{tabular}

a Agrupación de dicotomías. Tabulado el valor 1.

Por otra parte, y según las bases de datos consultadas, el 23,4\% de los textos no ha sido citado en ninguna ocasión, el 44,7\% tiene entre 1 y 5 citas, el $12,8 \%$ entre 6 y 10 , el 8,5\% entre 11 y 15 y un $10,6 \%$ de los textos ha tenido más de 15 citas.

\subsection{Elementos investigados}

El análisis de las características sociodemográficas (27,7\%), el estudio de los roles y/o las tareas de los trabajadores en la empresa (12,8\%) y la segregación vertical $(10,6 \%)$ son los temas que han acaparado preferentemente el interés en los textos recuperados (ver tabla 5). 
Tabla 5: tema de estudio de los artículos analizados*

\begin{tabular}{|c|c|c|c|}
\hline \multirow{2}{*}{ Objeto de estudio $^{a}$} & \multicolumn{2}{|c|}{ Respuestas } & \multirow{2}{*}{$\begin{array}{c}\text { Porcentaje } \\
\text { de casos }\end{array}$} \\
\hline & Frecuencia & Porcentaje & \\
\hline Características sociodemográficas & 26 & 27,7 & 92,9 \\
\hline $\begin{array}{l}\text { Roles y/o tareas que realizan los } \\
\text { trabajadores en la empresa }\end{array}$ & 12 & 12,8 & 42,9 \\
\hline Segregación vertical & 10 & 10,6 & 35,7 \\
\hline Retribuciones & 8 & 8,5 & 28,6 \\
\hline $\begin{array}{l}\text { Conflicto familia-trabajo y/o conflicto } \\
\text { trabajo-familia }\end{array}$ & 7 & 7,4 & 25 \\
\hline Estereotipos o creencias de género & 7 & 7,4 & 25 \\
\hline Actitudes hacia el trabajo/empresa & 7 & 7,4 & 25 \\
\hline Satisfacción laboral & 7 & 7,4 & 25 \\
\hline Segregación horizontal & 4 & 4,3 & 14,3 \\
\hline Estrés & 3 & 3,2 & 10,7 \\
\hline Uso de las nuevas tecnologías & 2 & 2,1 & 7,1 \\
\hline Medidas de igualdad & 1 & 1,1 & 3,6 \\
\hline TOTAL & 94 & 100 & 335,7 \\
\hline
\end{tabular}

a Agrupación de dicotomías. Tabulado el valor 1.

*Tabla de respuestas múltiples, un artículo puede tratar distintos temas

Más del $80 \%$ de los artículos se han centrado en estudiar organizaciones y/o trabajadores; únicamente en dos ocasiones la unidad de análisis ha sido diferente (artículos y estudiantes). En la mayoría de los casos se han investigado instituciones en Estados Unidos (15 de 29 artículos), dos casos se centran en Reino Unido y otros dos estudian un conjunto de diversos países europeos. El resto de zonas geográficas únicamente han sido consideradas en una ocasión (Emiratos Árabes Unidos, Taiwán, Holanda, Alemania, Indonesia y Rumania). Por otra parte, el $69 \%$ de los artículos ha observado a hombres y a mujeres, frente al $20,7 \%$ que únicamente ha analizado población femenina. Sólo el $6,9 \%$ se ha centrado en varones.

Un 48,3\% del total de los documentos recuperados analiza directa o indirectamente la discriminación por razón de sexo en las organizaciones, aunque solamente hemos encontrado un texto que define explícitamente los términos «sexismo» y/o «discriminación». 9 investigaciones concluyen que sí hay sexismo, 3 afirman que éste únicamente se produce en determinadas circunstancias y 2 sostienen que no existe. En un texto se señala que algunas mujeres 
aceptan su situación de discriminación para encajar y continuar en su puesto de trabajo (Wrigley 52).

\subsection{Metodología utilizada}

El 90\% de los artículos realizan investigaciones empíricas. De ellos, el 72,4\% utiliza la metodología cuantitativa y el $41,4 \%$ cualitativa, de forma que casi el $25 \%$ de las investigaciones combinan el uso de ambas. Cuando se recurre al análisis cuantitativo, las técnicas utilizadas son mayoritariamente la encuesta $(85,7 \%)$ y el análisis de contenido (14,3\%). Por su parte, los estudios cualitativos usan las entrevistas $(60 \%)$, los focus group $(33,3)$ y los análisis de discurso $(6,7 \%)$.

\subsection{Bibliografía y referencias utilizadas}

El 34,5\% de los textos recuperados cita entre 20 y 40 documentos y el 27,6\% entre 41 y 60. La gran mayoría de estos escritos están en inglés: únicamente tres artículos citan textos en otras lenguas (holandés, alemán, indonesio y malayo) (ver tabla 6).

Tabla 6: número de textos citados en las referencias bibliográficas de los artículos analizados

\begin{tabular}{|l|c|c|}
\hline \multicolumn{1}{|c|}{$\begin{array}{c}\text { Número de textos citados en las } \\
\text { referencias bibliográficas }\end{array}$} & Frecuencia & Porcentaje \\
\hline $\begin{array}{l}\text { Desconocido (no se ha podido obtener } \\
\text { esta información) }\end{array}$ & 1 & 3,4 \\
\hline Menos de 20 & 4 & 13,8 \\
\hline Entre 20 y 40 & 10 & 34,5 \\
\hline Entre 41 y 60 & 8 & 27,6 \\
\hline Entre 61 y 80 & 2 & 6,9 \\
\hline Más de 80 & 4 & 13,8 \\
\hline TOTAL & 29 & 100 \\
\hline
\end{tabular}

Los documentos incluidos en las referencias bibliográficas generalmente son citados en una única ocasión. Sin embargo, el escrito de Grunig, Toth y Hon aparece en 14 de los 29 artículos analizados y el de Cline, Toth, Turk, Walters, Johnson y Smith en 10. También hay dos artículos que son citados en 7 ocasiones (Aldoory, Toth; Toth, Serini, Wright, Emig). Estos dos últimos textos pertenecen a las dos revistas que mayor número de escritos han reportado en 
la realización de esta revisión sistemática, a la sazón, las más representativas en el ámbito de las relaciones públicas -Journal of Public Relations Research y Public Relations Review- (Kim, Choi, Reber, Kim 116).

\section{CONCLUSIONES}

Los artículos que se centran explícitamente en los profesionales de las relaciones públicas y la comunicación corporativa con enfoque de género son todavía escasos, ya que únicamente se han encontrado, y por tanto analizado, 29 artículos. Sin embargo, nuestra investigación apunta un reciente, aunque discreto, interés por el estudio de este ámbito laboral en diversas zonas geográficas. En este sentido, la dispersión de la investigación es grande y es difícil decir que existe un centro o un grupo de referencia. Pese a esta información, EE.UU es el país donde se ubican la mayoría de autores y donde se llevan a cabo la mayoría de estudios de modo que, en paralelo con lo que sucede en otros campos, los trabajadores, especialmente las trabajadoras de este país, han sido los más observados (Beurer-Zuellig, Fieseler, Meckel 271). Las bases de datos de referencia analizadas carecen de artículos en castellano sobre esta cuestión.

No existen todavía ni académicos ni instituciones especializadas en el análisis de la profesión del relaciones públicas y del profesional de la comunicación corporativa con enfoque de género, aunque una cantidad considerable de científicos en diversas partes del mundo han prestando atención al tema en algún momento. Han sido las mujeres quienes se han interesado mayoritariamente por el análisis de estas cuestiones, de modo que no sólo hay una feminización de las relaciones públicas (Chen 431; Simorangkir 26) sino también una feminización de la investigación sobre esta feminización. La falta de especialización, la dispersión y la sexualización de este campo de trabajo pueden considerarse aspectos positivos que garantizan la pluralidad, la transculturalidad y la motivación en estos trabajos (Míguez-González, BaamondeSilva, Corbacho-Valencia 9).

Más de la mitad de los textos han aparecido en revistas indexadas en las bases de datos JCR y SJR, lo que apunta a que, aunque el tema no sea habitual, aparece en revistas con relativa calidad. Esta calidad se ratifica con las citas, ya que los artículos seleccionados son mencionados en otros trabajos con frecuencia. Sin embargo, el impacto científico se ve relativamente limitado por el hecho de que el análisis de las estructuras de la comunicación con enfoque de género no es todavía un tema de agenda generalizado, aunque empieza a despertar el interés de científicos con capacidad de divulgar en revistas de calidad. 
Los textos se han publicado preferentemente en revistas enmarcadas en el campo de las relaciones públicas y la comunicación corporativa. Ninguno lo ha hecho en cabeceras especializadas en el análisis de género, esta situación podría indicar que no es un tema de interés para los estudios feministas. Aunque los comunicólogos se preocupan por el abordaje de las cuestiones relacionadas con las diferencias en función de sexo, los estudios de género no tienen entre sus objetos de análisis preferentes las estructuras de la comunicación corporativa.

La evaluación de la presencia de sexismo despierta interés, pero el concepto no es habitualmente ni definido ni operacionalizado en los textos, lo que puede conllevar que los investigadores estén considerando realidades diversas. Sin embargo, la realización de este estudio ha resultado de vital importancia, ya que se ha podido descubrir que en la mayoría de los textos se llega a la conclusión de que en las estructuras y en los procesos de las relaciones públicas y la comunicación corporativa se produce algún tipo de discriminación. En esta profesión feminizada, hay diferencias entre hombres y mujeres en la contratación, la promoción, la satisfacción laboral, las funciones y los salarios (Sha, Toth 93). En concreto, y aunque hay menos trabajadores masculinos que femeninos, ellos ostentan los altos cargos, ganan más y tienen más poder en las organizaciones (Pompper 86). Por tanto, estudiar la producción científica con enfoque de género en el ámbito laboral de las relaciones públicas y de la comunicación corporativa ha permitido conocer la existencia de sexismo en esta profesión, situación especialmente llamativa al considerar que se trata de un sector feminizado (Aldoory 670; Chen 431; Sha, Toth 93; Simorangkir 26; Wrigley 32).

Para finalizar, señalar que la principal limitación de este estudio se basa en haber analizado únicamente una muestra de la producción científica existente sobre el tema de estudio, ya que se han examinado los artículos publicados en revistas indexadas en las bases de datos Web of Science Core Collection, Scopus y Proquest Central. Por tanto, los resultados obtenidos deben considerarse únicamente como una tendencia. Así, una futura línea de trabajo sería analizar investigaciones difundidas en revistas incluidas en bases de datos diferentes. Además, y a la luz de la revisión realizada, nos proponemos aumentar la investigación en este ámbito, poniendo especial atención en la operacionalización de los conceptos y en el uso de indicadores.

\section{AGRADECIMIENTOS}

Las autoras desean agradecer al Punto Bibliotecario de Información al Usuario de la Universidad de Alicante (Punt BIU) y al servicio de préstamo interbibliotecario de la Universidad Jaume I su apoyo. 


\section{REFERENCIAS BIBLIOGRÁFICAS}

Aldoory, Linda y Elizabeth Lance Toth. «Gender Discrepancies in a Gendered Profession: A Developing Theory for Public Relations». Journal of Public Relations Research 14.2 (2002): 103-126.

Aldoory, Linda. «A (Re)conceived Feminist Paradigm for Public Relations: A Case for Substantial Improvement». Journal of Communication 55.4 (2005): 668-684.

Arnold, Mary Peterson, Marlene Lozada y Cynthia Linton. Women in Newspapers. How Much Progress Has Been Made. Ilianois: Media Management Center at Northwestern University, 2003.

Arnold, Mary Peterson y Cynthia Linton. Women in Newspapers. How Much Progress Has Been Made. Ilianois: Media Management Center at Northwestern University, 2002.

Arnold, Mary Peterson. Women in Newspapers. How Much Progress Has Been Made. Ilianois: Media Management Center at Northwestern University, 2001.

Beurer-Zuellig, Bettina, Christian Fieseler y Miriam Meckel. «A Descriptive Inquiry Into the Corporate Communication Profession in Europe». Public Relations Review 35.3 (2009): 270-279.

Botan, Carl H. y Maureen Taylor. «Public Relations: The State of the Field». Journal of Communication 54.4 (2004): 645-661.

Bowman, Bobbi. Newsroom Employment Census. Reston: American Society of newspaper Editors, 2006.

Castillo, Antonio y Jordi Xifra. «Investigación bibliométrica de las tesis doctorales españolas sobre relaciones públicas (1965-2005)». Anàlisi: Quaderns de Comunicació i Cultura 34 (2006):141-161.

Castillo, Antonio y M. ${ }^{a}$ Carmen Carretón. «Investigación en Comunicación. Estudio bibliométrico de las Revistas de Comunicación en España». Comunicación y Sociedad 23.2 (2010): 289-327.

Chen, Yi-Ning Katherine. «Social Capital, Human Capital, and Career Success in Public Relations in Taiwan». Chinese Journal of Communication 4.4 (2011): 430-449.

Cline, Carolyn Garrett, Elizabeth Lance Toth, Judy VanSlyke Turk, Lynne Masel Walters, Nancy Johnson y Hank Smith. The Velvet Ghetto: The Impact of the Increasing Percentage of Women in Public Relations and Business Communication. San Francisco: IABC Foundation, 1986.

Comisión de Derechos de la Mujer e Igualdad de Género del Parlamento Europeo. Informe sobre el Impacto del Marketing y la Publicidad en la Igualdad entre Mujeres y Hombres, documento de sesión, 29 de mayo de 2008, (2008/2038(INI)), 2008. 
Comisión de las Comunidades Europeas. Integrar la Igualdad de Oportunidades entre Hombres y Mujeres en el Conjunto de las Políticas y Acciones Comunitarias (COM(96) 67 final), 1996. <http://eur-lex.europa.eu/legal-content/ES/TXT/ $\mathrm{PDF} /$ ?uri=CELEX:51996DC0067\&from=EN>, consultado el 24-12-2015.

Comisión Europea. Manual para la Perspectiva de Género en las Políticas de Empleo, de Inclusión Social y de Protección Social. Luxemburgo: Oficina de Publicaciones Oficiales de las Comunidades Europeas, 2008.

De-Filippo, Daniela. «La producción científica española en Comunicación en WOS. Las revistas indexadas en SSCI (2007-12)». Comunicar 21.41 (2013): 25-34.

Desmond, Roger y Anna Danilewicz. «Women Are on, but not in, the News: Gender Roles in Local Television News». Sex Roles 62.10-11 (2010): 822-829.

Dominick, Joseph R. y Gail E Rauch. «The Image of Women in Network TV Commercials». Journal of Broadcasting 16.3 (1972): 259-265.

Döring, Nicola y Sandra Pöschl. «Images of Men and Women in Mobile Phone Advertisements: A Content Analysis of Advertisements for Mobile Communication Systems in Selected Popular Magazines». Sex Roles 55.3-4 (2006): 173-185.

Eisend, Martin. «A Meta-analysis of Gender Roles in Advertising». Journal of the Academy of Marketing Science 38.4 (2010): 418-440.

Espinar, Eva. «Estereotipos de género en los contenidos audiovisuales infantiles». Comunicar 15.29 (2007): 129-134.

Falk, Erika y Erin Grizard. The Glass Ceiling Persists: The Third Annual APPC Report on Women Leaders in Communication Companies. Pennsylvania: Annenberg Public Policy Center of the University of Pennsylvania, 2003.

Furnham, Adrian y Stephanie Paltzer. «The Portrayal of Men and Women in Television Advertisements: An Updated Review of 30 Studies Published Since 2000». Scandinavian Journal of Psychology 51.3 (2010): 216-236.

Gallego, Joana, Elvira Altés, María Eugenia Melús, Jaume Soriano y María José Cantón. «La prensa diaria por dentro: mecanismos de transmisión de estereotipos de género en la prensa de información general». Anàlisi: Quaderns de Comunicació i Cultura 28 (2002): 225-242.

García, Damián y Emilia Smolak-Lozano. «Comunicación de crisis: compilación y revisión de teorías y taxonomías prácticas desde una perspectiva cualitativa». Revista de comunicación Vivat Academia 15.124 (2013): 51-67.

García-González, Aurora y Teresa Piñeiro-Otero. «Las mujeres en el ámbito de la producción publicitaria. Estudio del sector publicitario gallego desde una perspectiva de género». Revista Latina de Comunicación Social 66 (2011): 505-525.

Gentry, James y Robert Harrison. «Is Advertising a Barrier to Male Movement Toward Gender Change?». Marketing Theory 10.1 (2010): 74-96. 
Gil, Eva María. «La publicidad radiofónica en la programación de las distintas franjas horarias. Reflexiones desde la perspectiva de género». Aposta. Revista de Ciencias Sociales 64 (2015): 1-34.

Golob, Ursa, Klement Podnar, Wim J. Elving, Anne Ellerup Nielsen, Christa Thomsen y Friederike Schultz. «CSR Communication: Quo Vadis?». Corporate Communications: An International Journal 18.2 (2013): 176-192.

Grunig, Larissa A., Elizabeth Lance Toth y Linda Childers Hon. Women in Public Relations: How Gender Influences Practice. New York: Guilford Press, 1991.

Hernández-Ruiz, Alejandra, Marta Martín-Llaguno y Marina Beléndez-Vázquez. «La representación de la mujer en publicidad: (Des)igualdad cuantitativa y cualitativa en la creatividad española». Estudios Sobre el Mensaje Periodístico 18.2 (2012): 521-530.

Hovland, Carl I., Arthur A. Lumsdaine y Fred D. Sheffield. Experiments on Mass Communication. Studies in Social Psychology in World War II. Vol. 3. Princeton, NJ: Princeton University Press, 1949.

ICCO. «World PR Report 2014». International Communications Consultancy Organisation and The Holmes Report, 2014. <http://worldreport.holmesreport. com>, consultado el 12-11-2014.

Kelly, Erin L., Phyllis Moen, J. Michael Oakes, Wen Fan, Cassandra Okechukwu, Kelly D. Davis...Lynne M. Casper. «Changing Work and Work-Family Conflict: Evidence from the Work, Family, and Health Network». American Sociological Review 1.32 (2014): 1-32.

$\mathrm{Ki}$, Eyun-Jung y Jae-Hwa Shin. «Status of Organization-Public Relationship Research from an Analysis of Published Articles, 1985-2004». Public Relations Review 32.2 (2006): 194-195.

Kim, Soo-Yeon, Myung-II Choi, Bryan H. Reber y Daewook Kim. «Tracking Public Relations Scholarship Trends: Using Semantic Network Analysis on PR Journals from 1975 to 2011». Public Relations Review 40.1 (2014): 116-118.

Kim, Sora, Elizabeth Johnson Avery y Ruthann W. Lariscy. «Are Crisis Communicators Practicing What We Preach?: An Evaluation of Crisis Response Strategy Analyzed in Public Relations Research from 1991 to 2009». Public Relations Review 35.4 (2009): 446-448.

Klein, Donna. Women in Advertising. Ten Years on. London: IPA, 2001.

Krohling, Margarida M. «Organizational Communication and Public Relations Prospects for Latin American Studies». Revista Internacional de Relaciones Públicas 1.1 (2011): 69-96.

Lasswell, Harold Dwight. The Analysis of Political Behavior: An Empirical Approach. New York: Oxford University Press, 1948.

Lauzen, Martha M. y David M.Dozier. «Maintaining the Double Standard: Portrayals of Age and Gender in Popular Films». Sex Roles 52.7-8 (2005): 437-446. 
Lazarsfeld, Paul Felix, Bernard Berelson y Hazel Gaudet. The People's Choice. New York: Duell, Sloan \& Pearce, 1944.

Macharia, Sarah, Dermot O'Connor y Lilian Ndangam. Who Makes the News? Global Media Monitoring Project 2010, 2010. <http://whomakesthenews.org/ gmmp/gmmp-reports/gmmp-2010-reports>, consultado el 24-12-2015.

Major, Virginia Smith, Katherine J. Klein y Mark G. Ehrhart. «Work Time, Work Interference with Family, and Psychological Distress». Journal of Applied Psychology 87.3 (2002): 427-436.

Marcos, Juan Carlos, María Jesús Martínez y María Francisca Blasco. «Producción y dirección de tesis doctorales sobre publicidad en la universidad española (1971-2010)». Revista Española de Documentación Científica 35.3 (2012): 433-452.

Martín-Llaguno, Marta. «La mujer en la industria publicitaria. La concentración horizontal en la comunicación comercial». Anàlisi: Quaderns de Comunicació i Cultura 35 (2007a): 95-136.

Martín-Llaguno, Marta. «La mujer en la industria publicitaria. La segregación vertical en la comunicación comercial: Techo de cristal y suelo pegajoso». Zer. Revista de Estudios de Comunicación 12.22 (2007b): 429-452.

Martín-Llaguno, Marta y Teresa Baquerín de Riccitelli. «Radiografía del sector publicitario Argentino. Características sociodemográficas, organizacionales y actitudinales de la fuerza laboral de la comunicación comercial». Ecos de la Comunicación 4 (2011): 75-92.

Martín-Llaguno, Marta, Marina Beléndez-Vázquez y Alejandra Hernández-Ruiz. La mujer en las agencias de publicidad: Categorías, especializaciones y conflicto trabajo-familia en las agencias españolas. Madrid: Asociación Española de Agencias de Publicidad, 2007.

Martín-Llaguno, Marta, Susana Miquel Segarra y Marián Navarro-Beltrá. «Analysis of Communication Executives and Managers in Spain: Socio-Demographic, Organizational and Attitudinal Characteristics from a Gender Perspective». Catalan Journal of Communication \& Cultural Studies 7.2 (2015): 129-146.

Martín-Llaguno, Marta y Marián Navarro-Beltrá. «Leyes de violencia de género y sexismo publicitario: análisis comparativo de Argentina, España, Estados Unidos y México». Revista Panamericana de Salud Pública 33.4 (2013): 280-286.

Martínez, María Jesús. «La producción científica de monografías españolas sobre publicidad». Ibersid 5 (2011): 71-80.

Messineo, Melinda J. «Does Advertising on Black Entertainment Television Portray More Positive Gender Representations Compared to Broadcast Networks?». Sex Roles 59.9-10 (2008): 752-764.

Míguez-González, María Isabel, Xosé Manuel Baamonde-Silva y Juan Manuel Corbacho-Valencia. «A Bibliographic Study of Public Relations in Spanish 
Media and Communication Journals, 2000-2012». Public Relations Review 40.5 (2014): 818-828.

Morton, Linda P. y Li-Yun Lin. "Content and Citation. Analysis of Public Relations Review». Public Relations Review 21.4 (1995): 337-349.

Naciones Unidas. La Declaración Universal de Derechos Humanos, 1948. <http:// www.un.org/es/universal-declaration-human-rights/index.html>, consultado el 24-12-2015.

Naciones Unidas. Declaración sobre la Eliminación de la Discriminación contra la Mujer, 1967. <http://daccess-dds-ny.un.org/doc/RESOLUTION/GEN/ NR0/239/40/IMG/NR023940.pdf?OpenElement>, consultado el 16-04-2013.

Naciones Unidas. Proclamación de Teherán, 1968. <http://www.icam.es/docs/ficheros/200407060006_6_8.pdf>, consultado el 28-12-2014.

Naciones Unidas. Convención sobre la Eliminación de Todas las Formas de Discriminación contra la Mujer, 1979. <http://www.un.org/womenwatch/daw/cedaw/ text/sconvention.htm>, consultado el 28-12-2015.

Navarro-Beltrá, Marián y Marta Martín-Llaguno. «A Systematic Review of Gender and Advertising Studies». Catalan Journal of Communication \& Cultural Studies 4.13 (2012): 171-183.

Navarro-Beltrá, Marián y Marta Martín-Llaguno. «Bibliometric Analysis of Research on Women and Advertising: Differences in Print and Audiovisual Media». Comunicar 21.41 (2013): 105-114.

Oliva, Carlos. «Visibilidad y producción científica de las Tesis Doctorales de Historia del Periodismo en las universidades españolas (2002-2012)». Historia y Comunicación Social 19.Número especial (febrero) (2014): 183-193.

Pasadeos, Yorgo y Bruce Renfro. «Research in Brief. A Citation Study of Public Relations Research, 1975-1986». Public Relations Review 15.8 (1989): 48-50.

Pasadeos, Yorgo, Bruce Berger y Bruce Renfro. «Public Relations as a Maturing Discipline: An Update on Research Networks». Journal of Public Relations Research 22.2 (2010): 136-158.

Pasadeos, Yorgo, Bruce Renfro y Mary Lynn Hanily. «Influential Authors and Works of the Public Relations Scholarly Literature: A Network of Recent Research». Journal of Public Relations Research 11.1 (1999): 29-52.

Pompper, Donnalyn. «On Social Capital and Diversity in a Feminized Industry: Further Developing a Theory of Internal Public Relations». Journal of Public Relations Research 24.1 (2012): 86-103.

Sallot, Lynne M., Lisa J. Lyon, Carolina Acosta-Alzuru y Karyn Ogata Jones. «From Aardvark to Zebra: A New Millennium Analysis of Theory Development in Public Relations Academic Journals». Journal of Public Relations Research 15.1 (2003): 27-90. 
Sha, Bey-Ling y Elizabeth L. Toth. «Future Professionals' Perceptions of Work, Life, and Gender Issues in Public Relations». Public Relations Review 31.1 (2005): 93-99.

Silverstein, Arthur J. y Rebecca Silverstein. «The Portrayal of Women in Television Advertising». Federal Communications Bar Journal 27.1 (1974): 71-98.

Simorangkir, Deborah. «The Impact of the Feminization of the Public Relations Industry in Indonesia on Communication Practice». International Journal of Strategic Communication 5.1 (2011): 26-48.

Toth, Elizabeth Lance, Shirley A. Serini, Donald K. Wright y Arthur G. Emig. «Trends in Public Relations Roles: 1990-1995». Public Relations Review 24.2 (1998): 145-163.

Tsai, Wanhsiu Sunny. «Family Man in Advertising? A Content Analysis of Male Domesticity and Fatherhood in Taiwanese Commercials». Asian Journal of Communication 20.4 (2010): 423-439.

Unesco. Gender-Sensitive Indicators for Media. Draft Framework of Indicators to Gauge Gender Sensitivity in Media Operations and Content, Intergovernmental Council of the International Programme for the Development of Communication (Twenty-Eighth Session). Paris: Unesco, 2012.

Unión Europea. Resolución del Consejo y de los representantes de los gobiernos de los Estados miembros reunidos en el seno del Consejo de 5 de octubre de 1995 sobre el Tratamiento de la Imagen de las Mujeres y de los Hombres en la Publicidad y en los Medios de Comunicación. Diario Oficial de las Comunidades Europeas, 10 de noviembre de 1995, Serie C, número 296, 15-16.

Unión Europea. Resolución sobre la Discriminación de la Mujer en la Publicidad. Diario Oficial de las Comunidades Europeas, 06 de octubre de 1997, Serie C, número 304, 60-63.

Unión Europea. Carta de los Derechos Fundamentales de la Unión Europea. Diario Oficial de la Unión Europea, 18 de diciembre de 2000, Serie C, número $346,1-22$.

Unión Europea. Versiones Consolidadas del Tratado de la Unión Europea y del Tratado Constitutivo de la Comunidad Europea. Diario Oficial de la Unión Europea, 29 de diciembre de 2006, Serie C, número 321, 1-331.

Valls-Fernández, Federico y José Manuel Martínez-Vicente. «Gender Stereotypes in Spanish Television Commercials». Sex roles 56.9-10 (2007): 691-699.

Van Zoonen, Liesbet. Feminist Media Studies. London: Sage, 1994.

Wrigley, Brenda J. «Glass Ceiling? What Glass Ceiling? A Qualitative Study of How Women View the Glass Ceiling in Public Relations and Communications Management». Journal of Public Relations Research 14.1 (2002): 27-55.

Ye, Lan y Eyun-Jung Ki. "The Status of Online Public Relations Research: An Analysis of Published Articles in 1992». Journal of Public Relations Review 24.5 (2012): 409-434. 